\title{
Continuous and Pulsed Quantum Zeno Effect
}

\author{
Erik W. Streed ${ }^{1,2}$, Jongchul Mun ${ }^{1}$, Micah Boyd ${ }^{1}$, Gretchen K. \\ Campbell $^{1}$, Patrick Medley ${ }^{1}$, Wolfgang Ketterle ${ }^{1}$, David E. Pritchard ${ }^{1}$ \\ ${ }^{1}$ Department of Physics, MIT-Harvard Center for Ultracold Atoms, \\ and Research Laboratory of Electronics, MIT, Cambridge, Massachusetts 02139, USA \\ ${ }^{2}$ Centre for Quantum Dynamics, Griffith University, Nathan, QLD 4111, Australia
}

(Dated: February 6, 2008)

\begin{abstract}
Continuous and pulsed quantum Zeno effects were observed using a ${ }^{87} \mathrm{Rb}$ Bose-Einstein condensate(BEC). Oscillations between two ground hyperfine states of a magnetically trapped condensate, externally driven at a transition rate $\omega_{R}$, were suppressed by destructively measuring the population in one of the states with resonant light. The suppression of the transition rate in the two level system was quantified for pulsed measurements with a time interval $\delta t$ between pulses and continuous measurements with a scattering rate $\gamma$. We observe that the continuous measurements exhibit the same suppression in the transition rate as the pulsed measurements when $\gamma \delta t=3.60(0.43)$, in agreement with the predicted value of 4 . Increasing the measurement rate suppressed the transition rate down to $0.005 \omega_{R}$.
\end{abstract}

PACS numbers: 03.65.Xp,03.75.Mn,42.50.Xa

The quantum Zeno effect (QZE) is the suppression of transitions between quantum states by frequent measurements. It was first considered as a theoretical problem where the continuous observation of an unstable particle would prevent its decay [1]. Experimental demonstrations of the QZE $[2,3,4,5,6,7,8]$ have been driven by interest in both fundamental physics and practical applications. Practical applications of the QZE include reducing decoherence in quantum computing [8, 9, 10], efficient preservation of spin polarized gases [3, 4, 6], and dosage reduction in neutron tomography [11].

The QZE is a paradigm and test bed for quantum measurement theory[12, 13]. In one interpretation, it involves many sequential collapses of the wavefunctions of the system. Quantum Zeno experiments provide constraints for speculative extensions of quantum mechanics where the collapse of the wavefunction is created by extra terms in a modified Schrödinger equation [14]. It is still an open question how close one can approach the limit of an infinite number of interrogations due to the Heisenberg uncertainty involved in shorter measurement times. These conceptional questions provide the motivation to extend experimental tests of the quantum Zeno phenonmenon. A major improvement to a quatum Zeno experiment with ultracold neutrons [15] is in preparation.

In this letter we compare the suppression of the transition rate in an oscillating two level system by continuous and pulsed measurements. Our QZE experiments were carried out with Bose-Einstein condensed atoms [16, 17, 18]. The long coherence time and the high degree of control of the position and momentum of the atoms created a very clean system and allowed us to observe much stronger quantum Zeno suppression than before [2, 5, 7]. In the experiment with pulsed measurements up to 500 measurements could be carried out and survival probabilities exceeded 98\%. Furthermore, we have performed the first quantitative comparison between the pulsed and continuous measurement QZE. This is important since any real pulsed measurement is only an approximation based on a series of weak continuous measurements [19, 20].

Let us consider a two-level system which is externally driven at a Rabi frequency $\omega_{R}$. Measurements of the state of the system project the system into one of the two states $|1\rangle,|2\rangle$. If the initial state of the system is in $|1\rangle$ and a measurement is made after short time $\delta t\left(\ll 1 / \omega_{R}\right)$, then the probability that the system is in $|1\rangle$ is $1-\left(\omega_{R} \delta t / 2\right)^{2}$. With $N$ successive measurements the probability that the system remains in $|1\rangle$ is

$$
\begin{aligned}
P(N) & =\left[1-\left(\omega_{R} \delta t / 2\right)^{2}\right]^{N} \approx \exp \left[-N\left(\omega_{R} \delta t / 2\right)^{2}\right] \\
& =\exp \left[-\left(\omega_{R}^{2} \delta t / 4\right) T\right]
\end{aligned}
$$

with $T=N \delta t$ the total free evolution time. Instead of normal Rabi-type oscillation between two states, the initial state $|1\rangle$ decays with an effective decay rate $1 / \tau_{E P}[21] .1 / \tau_{E P}$ is given by

$$
1 / \tau_{E P}=\omega_{R}^{2} \delta t / 4
$$

The characteristic time $\tau_{E P}$ for the pulsed QZE is much longer than the characteristic time $1 / \omega_{R}$ of normal Rabi-type oscillation. This shows the suppression of transition by the QZE.

For a continuous measurement, the atoms are continuously illuminated with laser light resonant with the transition energy between state $|2\rangle$ and another excited state. If atoms are in state $|2\rangle$, they spontaneously emit a photon at a rate $\gamma$. Due to the photon recoil, those atoms are removed from the coherently driven two-level system. The population of state $|1\rangle$ decays with the effective decay rate $1 / \tau_{E C}$ which is given by the optical Bloch equations as

$$
1 / \tau_{E C}=\omega_{R}^{2} / \gamma
$$

In contrast, for measurements with randomly spaced pulses, the effective decay rate is $1 / \tau_{E P}=\omega_{R}^{2}\left\langle\delta t^{2}\right\rangle / 4\langle\delta t\rangle$. If the 
(a)

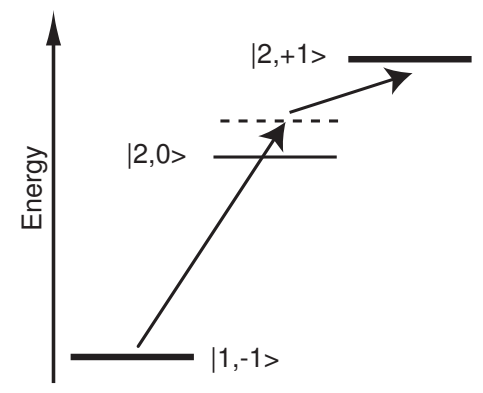

(b)
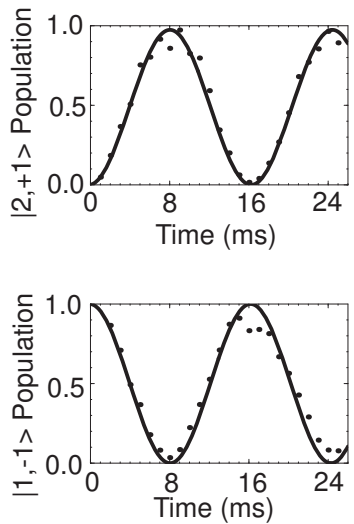

FIG. 1: Two-level Rabi oscillation. The two-level quantum system consisted of the $|1,-1\rangle$ and $|2,+1\rangle$ ground hyperfine states of ${ }^{87} \mathrm{Rb}$. a. Energy level diagram for relevant ${ }^{87} \mathrm{Rb}$ ground hyperfine states. Arrows depict the components of the two photon transition between the $|1,-1\rangle$ and $|2,+1\rangle$ states. $6.8 \mathrm{GHz}$ microwaves couple the $|1,-1\rangle$ to a virtual intermediate state detuned $420 \mathrm{kHz}$ above resonance with $|2,0\rangle$. Radio frequency (RF) at $1.68 \mathrm{MHz}$ resonantly completed the transition to the $|2,+1\rangle$ state. b. Driven population of the $|1,-1\rangle$ and $|2,+1\rangle$ states as a function of time. Curves are fits to a two photon transition rate of $\omega_{R} / 2 \pi=61.5(0.5) \mathrm{Hz}$. No population was detected in $|2,0\rangle$.

probability for measurement pulse during a time interval $\delta t$ is $\gamma \delta t,\left\langle\delta t^{2}\right\rangle=2 / \gamma^{2}$ and $\langle\delta t\rangle=1 / \gamma$. The effective decay rate for this case is

$$
1 / \tau_{E P, \text { random }}=\omega_{R}^{2} / 2 \gamma
$$

or twice the value of Eq. (3).

In our study we have determined the lifetimes $\tau_{E P}, \tau_{E C}$ with each type of measurement and used them to verify the prediction of Eq.(2), (3) that pulsed measurements with time interval $\delta t$ produce the same suppression of decay as continuous measurements with a scattering rate $\gamma$ when $\gamma \delta t=4[21]$. In particular, by verifying Eq. (3), we show that the continuous measurement process can not be simulated by a series of random pulses with a rate $\gamma$.

Our experimental system consisted of magnetically trapped ${ }^{87} \mathrm{Rb}$ Bose-Einstein condensate in the $5 \mathrm{~S}_{1 / 2}|1,-1\rangle\left(\left|F, m_{F}\right\rangle\right)$ and $5 \mathrm{~S}_{1 / 2}|2,+1\rangle$ states. Pure condensates of $N_{c}=$ $5.0(0.5) \times 10^{6}$ atoms in the $|1,-1\rangle$ state were prepared in a $\{63,63,6.6\} \mathrm{Hz}$ magnetic trap [22]. The atom number was then reduced to $N_{c}=5.0(0.5) \times 10^{4}$ by radio frequency $(\mathrm{RF})$ output coupling [23] to lower the density and collisional opacity. The lifetime of the reduced $|1,-1\rangle$ condensate exceeded $5 \mathrm{~s}$. During the experiments a RF shield maintained a magnetic trap depth of $5 \mu \mathrm{K}$. Coherent oscillations between state $|1\rangle(|1,-1\rangle)$ and state $|2\rangle(|2,+1\rangle)$ were then driven at a rate $\omega_{R}$ by a two photon transition (Fig. 11. The $|1,-1\rangle$ and $|2,+1\rangle$ states were selected because they have the same 1 st order Zeeman shift at a magnetic field of $3.23 \mathrm{G}$ [24].

Measurements of the population in state $|2\rangle(|2,+1\rangle)$ were performed by a laser beam of $780 \mathrm{~nm} \pi$ polarized light resonant with the $5 S_{1 / 2}|2,+1\rangle \rightarrow 5 P_{3 / 2}|3,+1\rangle$ transition. The

laser beam had a $1 / e^{2}$ diameter of $d_{0}=9.5(0.1) \mathrm{mm}$ and its power was monitored with a photodiode. The $362 \mathrm{nK}$ energy from a single photon recoil distinguished scattered atoms from the subrecoil $\mu=15 \mathrm{nK}$ energy range of the condensate atoms. Successive scatterings would eject measured atoms from the trap. After each QZE experiment was completed the magnetic trap was turned off and the population of surviving atoms in each state was measured. To simultaneously measure the $|1\rangle$ and $|2\rangle$ populations we used an RF pulse and magnetic field sweep to transfer the atoms to other magnetic sublevels. Parameters were chosen in such a way that each initial state was partially transferred to a sublevel with a different magnetic moment. After Stern-Gerlach separation and $41 \mathrm{~ms}$ of ballistic expansion, the atoms were imaged and the populations in the two initial states could be read out simultaneously [25].

We quantified the QZE induced by repeated pulsed measurements. Optical measurement pulses of $172 \mu \mathrm{W}\left(s_{0}=0.15\right.$, where $s_{0}=I / I_{\text {sat }}$ is the transition saturation parameter) and $t_{p}=10 \mu \mathrm{s}$ in duration were applied to the driven two level system. Each pulse scattered $\sim 29$ photons per atom and were separated by a free evolution time $\delta t$. The lifetime $\tau_{E P}$ for a particular measurement rate $1 / \delta t$ [29] was determined by fitting the $|1\rangle$ atom lifetime to an exponential decay curve over a range of times $\gtrsim 2 \tau_{E P}$. Fig. 2 a shows the dramatic increase in the observed lifetimes (solid symbols) as the measurement rate $1 / \delta t$ was increased. The measured lifetimes for two different $\omega_{R}$ ( boxes for $2 \pi \cdot 54.6(0.5) \mathrm{Hz}$, triangles for $2 \pi \cdot 24.7(0.1) \mathrm{Hz})$ are plotted along with their expected values (lower and upper lines respectively). The measured lifetimes were not found to be strongly sensitive to variations in optical power, pulse width, or laser detuning. The lifetime enhanced by QZE can be compared to $1 / \omega_{R}$, which would be the characteristic time without pulsed measurements. The longest lifetime was $198(16) \cdot 1 / \omega_{R}$ at $1 / \delta t=25 \mathrm{~ms}^{-1}$.

Previous works [2, 7, 26] express the QZE in terms of the survival probability $P(N)$ for number of measurements $N$ during a $\pi$ pulse $\left(t=\pi / \omega_{R}\right)$, a duration where without measurements $100 \%$ of the atoms would be transferred into the other state. Fig. 2b displays our results in this way. In these terms the greatest Zeno effect is for $\mathrm{N}=506(2)$ measurements with a survival probability $\mathrm{P}=0.984(1)$.

The most frequent measurements (farthest right solid symbols in Fig. 2a) show significant deviation from expected lifetimes(lines). For a high measurement rate $1 / \delta t$, the pulse duration $t_{p}$ is not negligible compared to free evolution time $\delta t$ between the pulses and the process that occurs while the measurement pulse is on becomes more important. In our experiment the pulse duration $t_{p}=10 \mu$ s was $20 \%$ of the shortest time interval $\delta t=40 \mu \mathrm{s}$. In such cases the measured lifetime depends not only on the time interval $\delta t$ but also on the pulse duration $t_{p}$. During the time interval, $\delta t$ the population in state $|1\rangle$ transfers("decays") to state $|2\rangle$ with $\tau_{E P}$. During the pulse duration $t_{p}$, state $|1\rangle$ can decay by different loss mechanisms. We made a separate measurement of this additional loss. The system was prepared in the same way except that the measurement pulse laser was kept on continuously. 

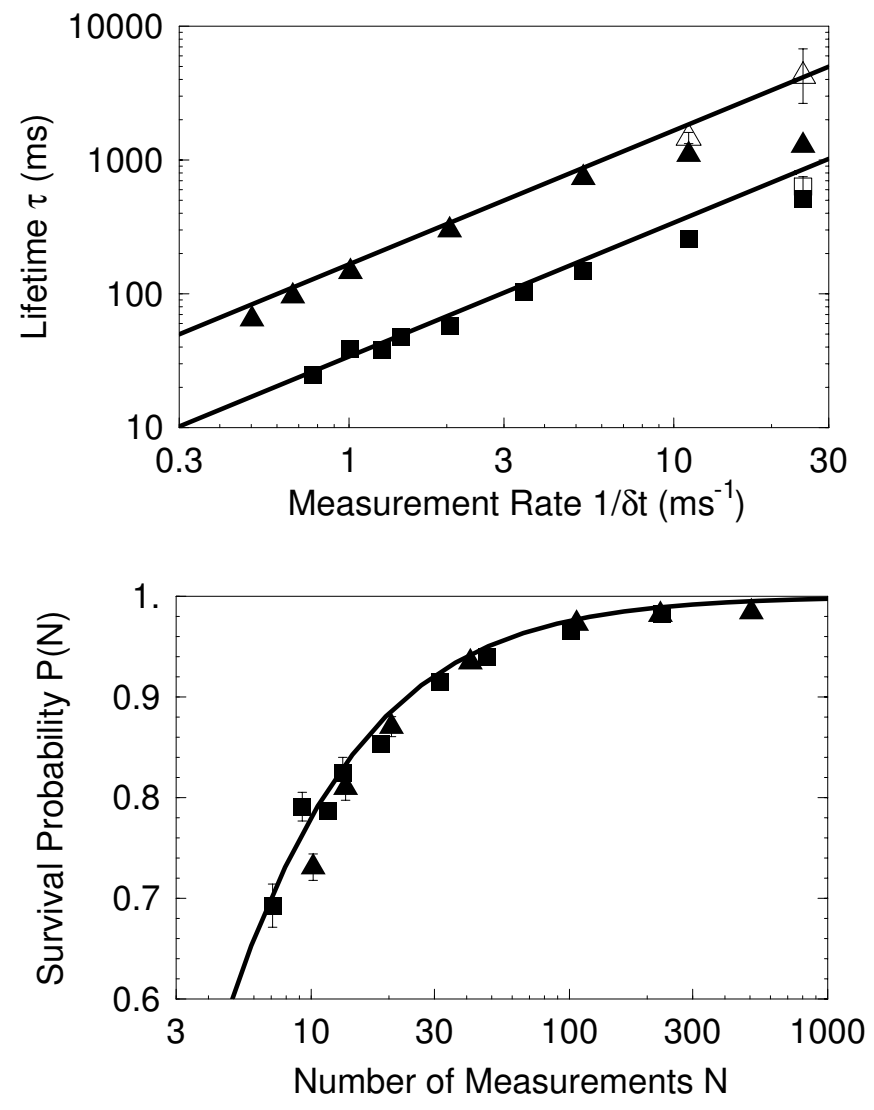

FIG. 2: Pulsed quantum Zeno effect. Increase in the lifetime (a) and the survival probability (b) of atoms in the initial $|1\rangle$ state as the measurement rate $1 / \delta t$ is increased. Solid lines indicate the prediction for the pulsed QZE. Boxes (triangles) are data points for a transition rate $\omega_{R} / 2 \pi=54.6(0.5)(24.7(0.1)) \mathrm{Hz}$. a. Observed lifetimes (Solid) for $|1\rangle$ atoms measured with a time interval $\delta t$ between measurement pulses. Lines indicate the expected QZE lifetime $\tau_{E P}=4 /\left(\omega_{R}^{2} \delta t\right)$. Open symbols show lifetimes after correction for additional loss mechanism by Eq. 5 b. The same data is displayed in terms of the survival probability for $N$ measurements performed during a $\pi$ pulse time $t=\pi / \omega_{R}$ ( the time which would take to transfer $100 \%$ of the atoms from $|1\rangle$ to $|2\rangle$ without measurements). The solid line is the expected survival probability $P(N)=\left[\cos \left(\frac{\pi}{2 N}\right)\right]^{(2 N)}$ for $\mathrm{N}$ ideal measurements.

The lifetime $1 / \Gamma_{m}$ of this system was measured and $\Gamma_{m}$ was $3.41(0.14) s^{-1}$ for $\omega_{R} / 2 \pi=54.6 \mathrm{~Hz}\left(\Gamma_{m}=2.96(0.22) s^{-1}\right.$ for $\left.\omega_{R} / 2 \pi=24.7 \mathrm{~Hz}\right)$. In order to find the origin of this additional loss, the measurements of lifetimes were made with removal of either the RF or the microwave component of the two-photon drive. The lifetime showed no change when the $\mathrm{RF}$ component was removed, but the lifetime increased by an order of magnitude without microwave component. This suggests that the loss occuring during pulse duration $t_{p}$ is dominated by the virtual intermediate state $|2,0\rangle$, which can be excited by the measurement laser to the excited state $5 P_{3 / 2}|3,0\rangle$

To obtain the correct decay rate $1 / \tau_{E P}$ for the pulsed QZE from our measurement this additional loss should be corrected for. The observed decay rate $1 / \tau$ is split into two components

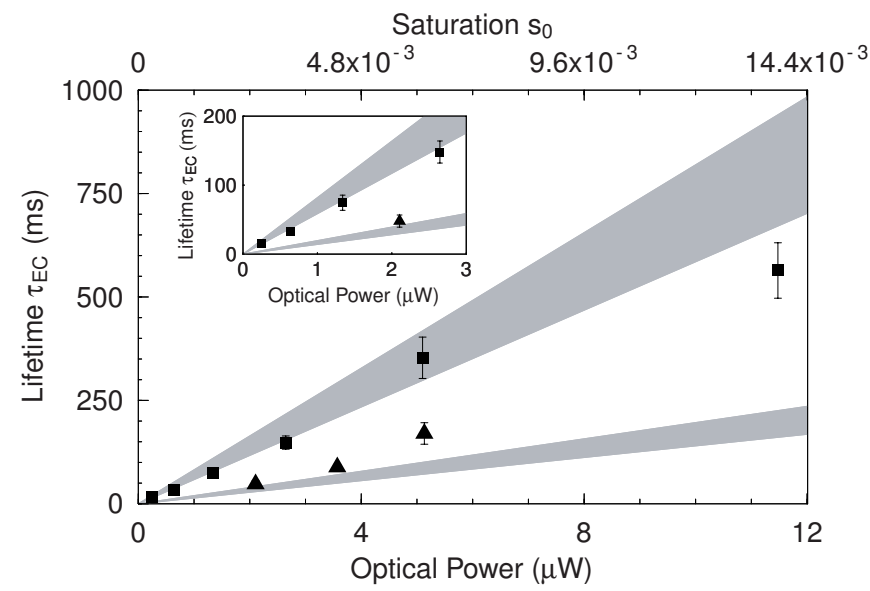

FIG. 3: Continuous quantum Zeno effect. Lifetime dependence on optical power with $\omega_{R} / 2 \pi=48.5(0.9) \mathrm{Hz}$ for laser detuning $\delta_{L}=$ $0 \mathrm{MHz}$ (boxes) and $\delta_{L}=-5.4 \mathrm{MHz}$ (triangles). Grey bands indicates range of expected lifetimes which are calculated from measurements of AC Stark shift for $\delta_{L}=0 \mathrm{MHz}$ (upper) and $\delta_{L}=-5.4$ $\mathrm{MHz}$ (lower). Inset highlights data from lower optical powers. The saturation parameter $s_{0}$ has an uncertainty of $17 \%$.

and can be written as

$$
\frac{1}{\tau}=\frac{1}{\tau_{E P}} \frac{\delta t}{t_{p}+\delta t}+\Gamma_{m} \frac{t_{p}}{t_{p}+\delta t}
$$

where $t_{p}$ is pulse duration. Data points in Fig. 2a where this correction had a significant impact on the lifetime are indicated by open symbols. The predicted lifetime is $\tau_{E P}=4 /\left(\omega_{R}^{2} \delta t\right)$, slightly larger than the measured $\tau_{E P}=$ $0.836(0.014) \times 4 /\left(\omega_{R}^{2} \delta t\right)$. The discrepancy is possibly due to collisions between recoiling atoms and the remaining condensate leading to additional loss.

The same initial system was subjected to a weak continuous measurement instead of repeated strong measurements. Fig. 3 shows the increase in lifetime with increasing measurement laser power. While showing this qualitative relationship is straightforward, several issues complicate a quantitative measurement of the continuous QZE. If the measurement laser is detuned from the optical resonance it will have both a reduced scattering rate and also induce an AC Stark shift $\delta_{R F}$ in the resonance between $|1\rangle$ and $|2\rangle$, reducing the effective Rabi frequency. In addition, imperfections in the beam can affect the intensity at the atoms. These issues are not important for the pulsed measurement as long as atoms scatter multiple photons. However they are critical to properly characterizing the weak continuous measurement experiment.

We were able to address all of these issues simultaneously by measuring the AC Stark shift at several different laser detunings. For each laser detuning $\left(\delta_{L}\right)$ and optical power $\left(s_{0}\right)$ we determined the AC Stark shift $\delta_{R F}$ by maximizing the reduction of atoms in state $|1\rangle$ as a function of RF frequency. Measurements of continuous QZE lifetime $\tau_{E C}$ (Eq. 3 ) were then made varying saturation parameter $s_{0}$ and detuning $\delta_{L}$ of 


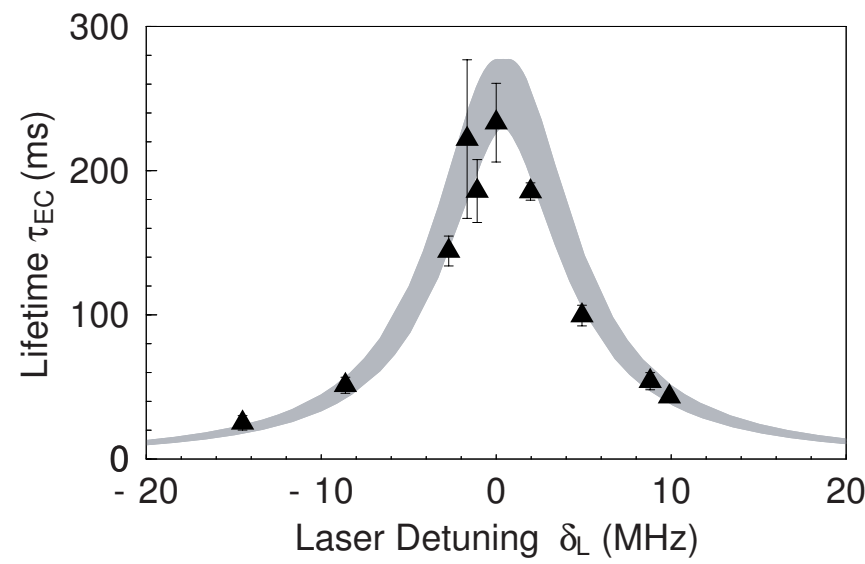

FIG. 4: Continuous quantum Zeno lifetime as a function of the measurement laser detuning $\delta_{L}$. Grey band indicates range of expected lifetimes(Eq. 6 from separately measured AC Stark shift parameters. The predicted linewidth is slightly Zeeman broadened by imperfections in the polarization. Data is for $3.5 \mu \mathrm{W}$ laser power, $\omega_{R}=45.5(1.0) \mathrm{Hz}$.

the measurement laser. Eq. 3 can then be rewritten as

$$
\tau_{E C}=\frac{\gamma}{\omega_{R}^{2}}=\frac{\Gamma s_{0}}{2 \omega_{R}^{2}}\left(\frac{1}{1+4\left(\frac{\delta_{L}}{\Gamma}\right)^{2}}\right)
$$

which is a function of $s_{0}$ and $\delta_{L}$ with ${ }^{87} \mathrm{Rb} \mathrm{D}_{2}$ transition decay rate $\Gamma$. Fig. (4verifies Eq. (6) for various detunings $\delta_{L}$. Fig. 3 shows increasing lifetime with increasing measurement laser power, the signature of the continuous QZE. Similar to the longest lifetime point in the pulsed QZE data (upper right solid triangle, Fig. [2), the data point with highest power in Fig. 3 shows significant deviation from the lifetime expected from Eq. 6 By matching the observed lifetimes for pulsed and continuous QZE measurements we find that each measurement type has the same QZE when $\gamma \delta t=3.60(0.43)$, which is in agreement with the predicted ratio of 4 [21] but rules out randomly repeated pulse case in Eq. (4). Eq. (4) gives the ratio of 2 instead of 4 . The observed large quantum Zeno suppression dramatically illustrates the modification of a wavefunction by a null measurement, i.e. the observation that no light has been scattered[27]. The large fraction of atoms in the initial state $|1\rangle$ is caused by repeated measurements without scattering any photons.

We have extended previous work in pulsed QZE measurements [2, 5, 7] by exploiting advantages inherent to BoseEinstein condensates. While in theory the Heisenberg uncertainty principal limits how frequently meaningful measurements can be performed, in practice imperfections in real measurements are the limiting factors [26, 28]. In ion experiments optical pumping between states during the measurement pulses changed the observed population transfer [2], requiring significant corrections for the $\mathrm{N}=32$ and $\mathrm{N}=64$ pulse measurements (Table I, [2]) to observe a maximum survival probability $P(64)=0.943(20)[2]\left(\tau_{E P}=54(30) \cdot 1 / \omega_{R}\right)$.
Previous demonstrations of the continuous QZE [3, 4, 6] observed qualitative, but not quantitatively characterized QZE suppression effects up to $80 \%$ [4] with increasing laser intensity. Our observed quantum Zeno suppressions are substantially larger then both previous pulsed [2] and continuous [4] results, and is also greater then that expected from proposed experiments [11, 15, 26, 28] in neutrons.

In conclusion we have used a Bose-Einstein condensate to demonstrate the QZE for both continuous and pulsed measurements. Lifetimes for both cases were substantially enhanced by QZE to values close to $200 \cdot 1 / \omega_{R}$ Pulsed and continuous QZE were quantified and compared. We observe that the continuous measurements exhibit the same suppression in the transition rate as the pulsed measurements when $\gamma \delta t=3.60(0.43)$, which agrees with the predicted value of 4 [21] and rules out a simple model when a continuous measurement is replaced by a series of random pulses. A next generation experiment could demonstrate even stronger quantum Zeno suppression and study the transition from pulsed to continuous QZE by using pulse duration and intervals approaching the spontaneous emission time.

The authors thank Helmut Rauch for insightful discussion. This work was supported by NSF.

[1] B. Misra and E. C. G. Sudarshan, J. Math. Phys. 18, 756 (1977).

[2] W. Itano, D. Heinzen, J. Bollinger, and D. Wineland, Phys. Rev. A 41, 2295 (1990).

[3] B. Nagels, L. J. F. Hermans, and P. L. Chapovsky, Phys. Rev. Lett. 79, 3097 (1997).

[4] K. Mølhave and M. Drewsen, Phys. Lett. A 268, 45 (2000).

[5] M. C. Fischer, B. Gutiérrez-Medina, and M. G. Raizen, Phys. Rev. Lett. 87, 040402 (2001).

[6] T. Nakanishi, K. Yamane, and M. Kitano, Phys. Rev. A 65, 013404 (2001).

[7] C. Balzer, T. Hannemann, D. Reib, C. Wunderlich, W. Neuhauser, and P. E. Toschek, Opt. Commun. 211, 235 (2002).

[8] O. Hosten, M. T. Rakher, J. T. Barreiro, N. A. Peters, and P. G. Kwiat, Nature 439, 949 (2006).

[9] J. D. Franson, B. C. Jacobs, and T. B. Pittman, Phys. Rev. A 70, 062302 (2004).

[10] P. Facchi, S. Tasaki, S. Pascazio, H. Nakazato, A. Tokuse, and D. A. Lidar, Phys. Rev. A 71, 022302 (2005).

[11] P. Facchi, Z. Hradil, G. Krenn, S. Pascazio, and J. Rehacek, Phys. Rev. A 66, 012110 (2002).

[12] K. Koshino and A. Shimizu, Phys. Rep. 412, 191 (2005).

[13] D. Home and M. A. B. Whitaker, Annals of Physics 258, 237 (1997).

[14] S. L. Adler, Phys. Rev. D 67, 025007 (2003).

[15] M. R. Jaekel, E. Jericha, and H. Rauch, Nuc. Inst. Meth. A 539, 335 (2005).

[16] M. H. Anderson, J. R. Ensher, M. R. Matthews, C. E. Wieman, and E. A. Cornell, Science 269, 198 (1995).

[17] K. B. Davis, M.-O. Mewes, M. R. Andrews, N. J. van Druten, D. S. Durfee, D. M. Kurn, and W. Ketterle, Phys. Rev. Lett. 75, 3969 (1995).

[18] C. C. Bradley, C. A. Sackett, J. J. Tollett, and R. G. Hulet, Phys. 
Rev. Lett. 75, 1687 (1995), ibid. 79, 1170 (1997).

[19] T. A. Burn, Am. J. Phys 70, 719 (2002).

[20] C. M. Caves and G. J. Milburn, Phys. Rev. A 36, 5543 (1987).

[21] L. Schulman, Phys. Lett. A 57, 1509 (1998).

[22] E. W. Streed, A. P. Chikkatur, T. L. Gustavson, M. Boyd, Y. Torii, D. Schneble, G. K. Campbell, D. E. Pritchard, and W. Ketterle, Rev. Sci. Instr. 77, 023106 (2006).

[23] M.-O. Mewes, M. R. Andrews, D. M. Kurn, D. S. Durfee, C. G. Townsend, and W. Ketterle, Phys. Rev. Lett. 78, 582 (1997).

[24] D. Harber, H. Lewandowski, J. McGuirk, and E. Cornell, Phys. Rev. A 66, 053616 (2002).
[25] E. W. Streed, Ph.D. thesis, Massachusetts Institute of Technology (2006), http://cua.mit.edu/ketterle_group/Theses/Streed thesis.pdf.

[26] P. Facchi, Y. Nakaguro, H. Nakazato, S. Pascazio, M. Unoki, and K. Yuasa, Phys. Rev. A 68, 012107 (2003).

[27] R. H. Dicke, Am. J. Phys. 49, 925 (1981).

[28] H. Rauch, Physica B 297, 299 (2001).

[29] Including short pulse duration $t_{p}$, the pulse repetition rate in the experiment was $1 /\left(\delta t+t_{p}\right)$ 